\title{
Is transcranial colour Duplex flow imaging of use in selection of patients with acute stroke for thrombolysis?
}
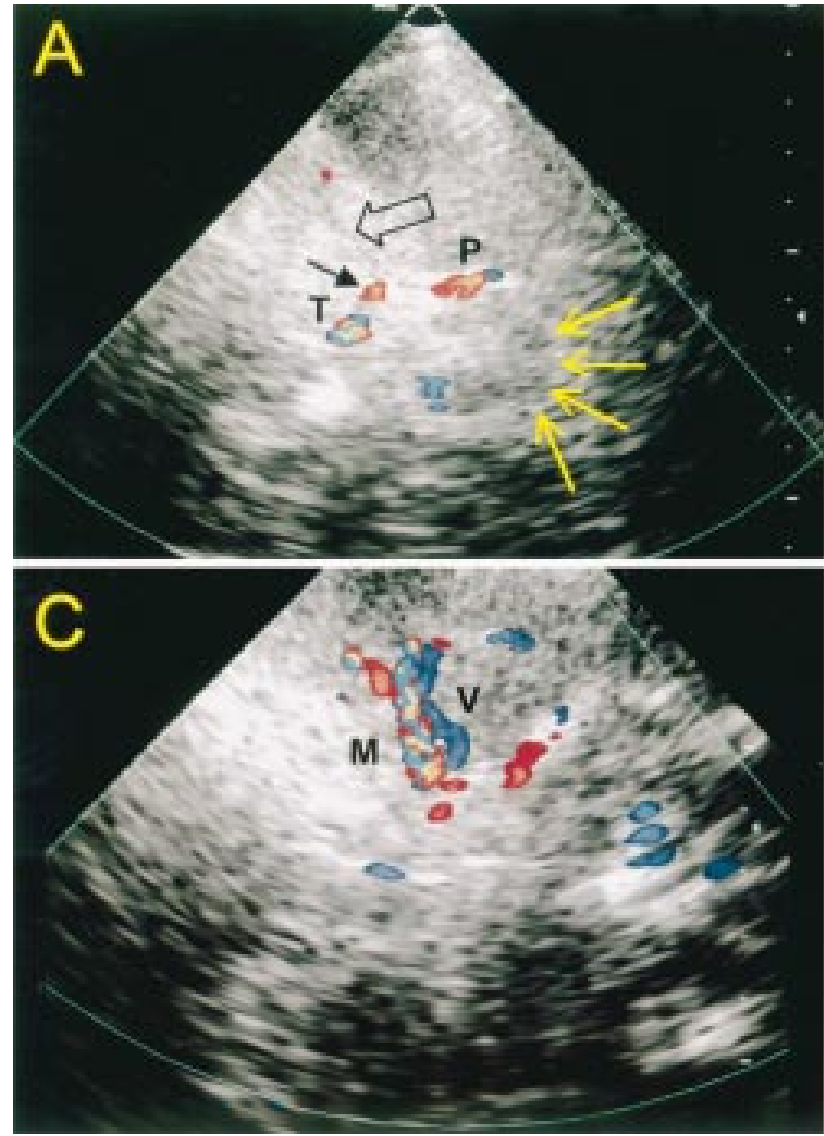

A 55 year old woman with an infarction in the left middle cerebral artery (MCA) territory, was investigated by transcranial colour Duplex flow imaging (TCDFI) in the emergency room 130 minutes after stroke onset, diagnosing a left MCA occlusion (figure A) confirmed by angiography (figure B). After an intra-arterial thrombolysis with infusion of 1.25 million units urokinase over 1.5 hours, a recanalisation of the left MCA M1 and partially of M2 segments was visible both on TCDFI (figure C) and control angiography (figure D). The immediate neurological recovery was good with a mild disability present at discharge. (A) TCDFI before thrombolysis showing occlusion of the left MCA (open arrow) just after the origin of the M1 segment (small arrow). T denotes the terminal portion of the internal carotid artery and $\mathrm{P}$ the posterior cerebral artery. The mesencephalon is indicated by long arrows. (B) Angiography before thrombolysis showing occlusion of the left MCA just after the origin of the M1 segment (open arrow). (C) TCDFI 3 hours after thrombolysis demonstrating patent left MCA-M1 and M2 (M) with moderately accelerated blood flow velocities (PSV $=145 \mathrm{~m} / \mathrm{s}$ ). V denotes the prominent left insular vein. (D) Angiography after thrombolysis showing recanalisation of the left MCA-M1 segment and the anterior superficial branches of the left MCA (open arrow), while the posterior superficial branches remain occluded.

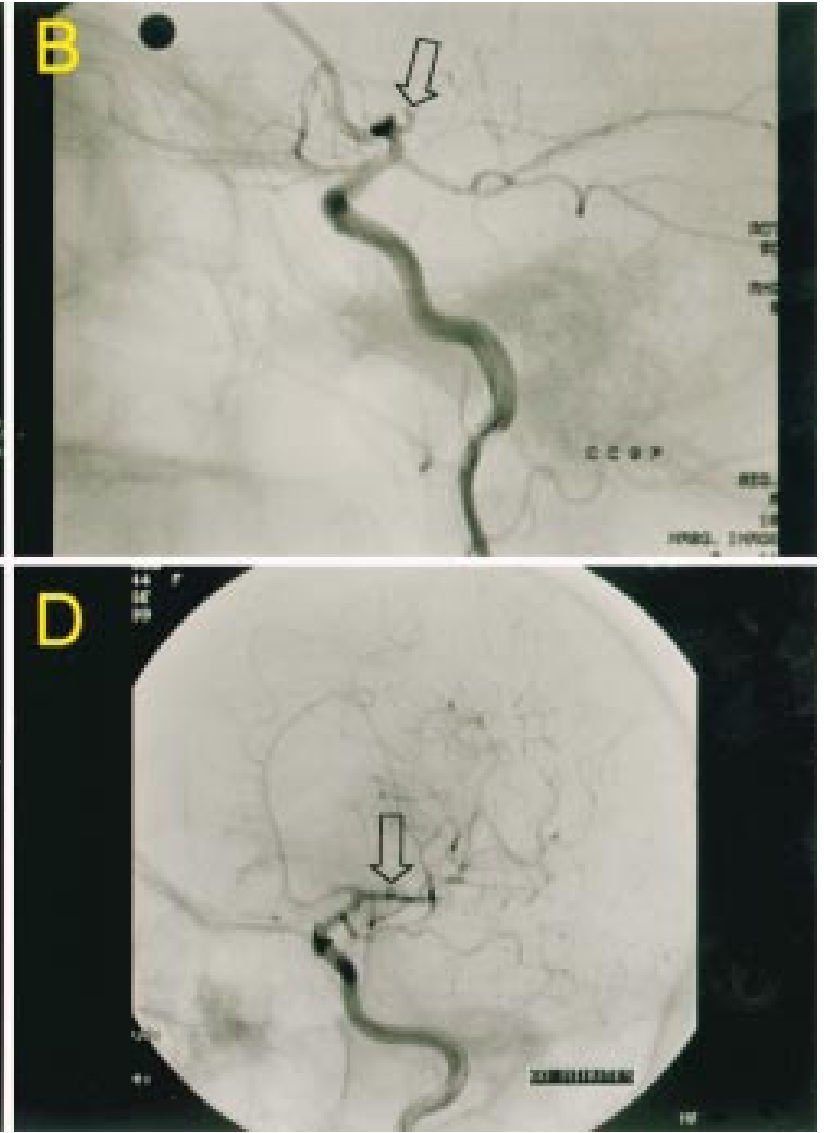

Thrombolysis is a potentially effective treatment in acute ischaemic stroke, but it is currently impossible to determine the risk to benefit ratio in individual patients, necessitating the definition of more precise tools for patient selection before its widespread adoption in clinical practice. An alternative technique to MRI, which still requires a major reorganisation of the emergency room, ${ }^{1}$ for identifying the presence of an occluded MCA could be TCDFI. It is noninvasive, not time consuming, relatively cheap, easy to perform by a well trained neurologist in the emergency room context, and obviously effective in depicting occlusion of the MCA $(\mathrm{M} 1 / \mathrm{M} 2){ }^{2}$ The potential role of TCDFI in patient selection for thrombolysis in an acute stroke setting has been recently suggested by some authors ${ }^{34}$ and warrants controlled studies, to be fully assessed.

GÉRALD DEVUYST NAZIRE AFSAR

Centre Hospitalier Universitaire Hospitalier Vaudois, Service de

Neurologie, BH-07, Rue du Bugnon 46, 1011 Lausanne, Switzerland

Correspondence to: Dr Gérald Devuyst

Gerald.Devuyst@chuv.hospvd.ch

1 Zivin JA. Thrombolytic stroke therapy: past, present, and future. Neurology 1999;53:14-19.

2 Markus HS. Transcranial Doppler ultrasound. $f$ Neurol Neurosurg Psychiatry 1999;67:135-7.

3 Kaps M, Link A. Transcranial sonographic monitoring during thrombolytic therapy. AfNR Am f Neuroradiol 1998;19:758-60.

4 Postert T, Braun B, Meves S, et al. Contrast-enhanced transcranial color-coded sonography in acute hemispheric brain infarction. Stroke 1999;30:1819-26. 